\title{
Senegalesische Corona-Songs als Sensibilisierungs- und Informationsquelle für wolofsprachige Geflüchtete und Migrant*innen
}

\section{Zusammenfassung}

Während der Corona-Pandemie ist der Zugang zu verlässlichen Informationen besonders relevant, um Schutzmaßnahmen ergreifen zu können. Migrant*innen und Geflüchteten in Transit- und Residenzländern mangelt es häufig am Zugang zu diesen Informationen. Dies öffnet den Raum für die Entstehung alternativer Sensibilisierungs- und Informationsquellen. Dazu zählen etwa Corona-Songs, also Lieder zur Sensibilisierung über COVID-19 und die nötigen Schutzmaßnahmen. Auch im Senegal sind seit Mitte März 2020 solche Lieder entstanden, die über soziale Medien weite Verbreitung finden. In diesem Artikel werden 20 senegalesische Corona-Songs und die dazugehörigen Videoclips auf ihr Potenzial als Sensibilisierungs- und Informationsquelle sowohl für die wolofsprachige Bevölkerung im Senegal als auch Migrant*innen und Geflüchtete in Transit- und Residenzländern analysiert.

Schlagworte: Corona-Songs, Senegal, Information, Sensibilisierungsmaßnahmen, Migration, Flucht, Corona, COVID-19

\section{Senegalese Corona Songs as a Source of Raising Awareness and Information for Wolof-speaking Refugees and Migrants}

\section{Abstract}

During the Corona pandemic, access to reliable information is particularly relevant in order to be able to take protective measures. However, migrants and refugees in transit and residence countries often lack such access to information. This opens the opportunity for alternative sources of raising awareness and information. Corona Songs about COVID-19 and the necessary protective measures constitute one of these alternatives. Such songs have been created in Senegal since mid-March 2020 and are widely distributed through social media. This article 
analyzes 20 Senegalese Corona Songs and their video clips for their potential as sources of raising awareness and information for both the Wolof-speaking population in Senegal and migrants and refugees in transit and residence countries.

Keywords: Corona Songs, Senegal, information, awareness raising measures, migration, refugees, Corona, COVID-19

\section{Einleitung}

Das Coronavirus SARS-CoV-2 verbreitet sich seit dem Auftreten der ersten Fälle im chinesischen Wuhan im Dezember 2019 auf der ganzen Welt. Insbesondere seit März 2020 ergreifen Regierungen diverse Maßnahmen, um die Ausbreitung in den jeweiligen Staaten zu verlangsamen und die Bevölkerung zu schützen. Hierzu zählen etwa Distanz- und Hygieneregeln, komplette Lockdowns mit Ausgangssperren sowie Grenzschließungen. Der Zugang zu verlässlichen Informationen über Entwicklungen und Sicherheitsmaßnahmen ist während der Corona-Pandemie höchst relevant, denn nur durch diese können sich die Menschen vor einer Ansteckung schützen. Doch die Möglichkeiten, an zuverlässige Informationen zu kommen, sind nicht für alle Menschen gleich. Alphabetisierung, Sprachkenntnisse und ein gewisser Bildungsstand wie auch der technische Zugang zu Informationen etwa über Fernsehen, Radio, Zeitungen oder Internet sind dafür wichtige Grundlagen. Besonders Geflüchteten und Migrant*innen in Transit- und Residenzländern fehlen mitunter diese Voraussetzungen (UNESCO 2018; Casey 2020; Refugees International 2020).

Dieser Artikel argumentiert, dass Corona-Songs, also Lieder, die im Laufe der Pandemie insbesondere in vielen afrikanischen Staaten entstanden sind, die Möglichkeit bieten, notwendige und verlässliche Informationen $\mathrm{zu}$ vermitteln (vgl. Fischer 2020). Diese Lieder sind zumeist vorrangig für die Sensibilisierung der Bevölkerung in den jeweiligen Entstehungsländern gedacht, erreichen aber durch soziale Medien und Youtube eine große Verbreitung. Sie dienen ferner Menschen auf der Flucht und auch in Residenzländern als Informationsquelle über das Virus und die empfohlenen Schutzmaßnahmen. Auch UNHCR (2020) weist auf den Nutzen von Liedern zur Sensibilisierung von Geflüchteten während der Pandemie hin. Obwohl vereinzelte Regierungen diese Methode zur Informationsverbreitung nutzen, sind Corona-Songs primär als Bottom-Up-Initiativen verschiedener Musiker*innen entstanden.

Anhand senegalesischer Corona-Songs widmet sich dieser Beitrag ihrem Potenzial als Sensibilisierungs- und Informationsquelle für die Bevölkerung des 
Senegals und besonders für wolofsprachige ${ }^{1}$ Geflüchtete und Migrant*innen in Transit- und Residenzländern. Die Lieder und die dazugehörigen Musikvideos werden dahingehend untersucht, welche Informationen und Erklärungen zum Coronavirus, zu den Schutzmaßnahmen und Verhaltensregeln und zur möglichen Dekonstruktion von $>$ Fake News bereitgestellt werden. Auch in vielen anderen afrikanischen Ländern sind Corona-Songs entstanden ${ }^{2}$, sodass das Phänomen der Corona-Songs nicht als spezifisch für den Senegal, sondern als weitreichend wirkend verstanden werden kann.

\section{Ungleicher Zugang zu Informationen in der Corona-Pandemie und Lieder als alternative Informationsquellen}

In der Corona-Pandemie sind Geflüchtete und Migrant*innen sowohl wegen der erschwerten Bedingungen bei der Einhaltung von Schutzmaßnahmen als auch wegen der Informationsknappheit in einer besonders prekären und vulnerablen Lage. Ungenügende Sprachkenntnisse, die Verbreitung von Falschinformationen, das geringe Vertrauen in lokale Autoritäten und fehlende Kommunikationsnetzwerke sind einige Gründe für diesen Informationsmangel (Refugees International 2020).

Der Norwegian Refugee Council fordert daher auf nationaler wie auch internationaler Ebene den »Zugang zu akkuraten und relevanten Informationen in geeigneten Sprachen« (Høvring 2020, eigene Übersetzung) für Menschen auf der Flucht sicherzustellen. Zudem betonen die Vereinten Nationen, dass leicht verständliche Sensibilisierungsmaßnahmen nötig sind, um über die Gefahren von und Schutzmaßnahmen zu COVID-19 aufzuklären (United Nations 2020). Zur Verbreitung offizieller Informationen verwenden viele Regierungschef*innen momentan Fernsehansprachen, welche jedoch hauptsächlich die eigene Bevölkerung erreichen.

Ein Versuch, dem Informationsmangel bei Geflüchteten und Migrant*innen entgegen zu wirken, sind die von Doctors of the World und dem Britischen Roten Kreuz herausgegebenen Informationsblätter in 51 verschiedenen Sprachen. Auch Wolof ist eine dieser Sprachen (Doctors of the World und British Red Cross

1 Wolof ist die Verkehrssprache des Senegals und wird im Gegensatz zur Amtssprache Französisch von fast allen Senegales*innen gesprochen. Auch in den Nachbarländern ist Wolof verbreitet, allerdings in viel geringerem Ausmaß.

2 Als Beispiele sind hier die kongolesischen Musiker*innen Koffi Olomide und Fally Ipupa, der ugandische Musiker und Oppositionspolitiker Bobi Wine, Hussein Masimbi aus Tansania und der südafrikanische Ndlovu Youth Choir zu nennen (Fischer 2020; Uimonen 2020). 
2020). Zwei Tatsachen schränken die Reichweite dieser schriftlichen Informationsquellen jedoch maßgeblich ein: Erstens müssen Menschen lesen können, um die Informationsblätter zu verstehen, und zweitens sind viele Menschen - besonders in afrikanischen Ländern - nicht in ihren jeweiligen Muttersprachen, sondern in den Ex-Kolonialsprachen alphabetisiert, weshalb Wolof in der Regel nicht gelesen werden kann. Der intendierte Mehrwehrt wolofsprachiger Informationsblätter ist also marginal und französischsprachige Informationen für alphabetisierte Senegales*innen nützlicher als ihre Übersetzung auf Wolof.

Auch Poster und Tafeln mit bildlichen Darstellungen von Schutzmaßnahmen werden ebenso wie Street-Art und Graffiti zur Aufklärung verwendet. Diese Art der Sensibilisierung ist leichter verständlich, jedoch nur lokal zugänglich, wodurch ihre Tragweite eingeschränkt ist. Fernsehserien und kurze Videoclips, die besonders während des Ramadans in vielen muslimisch geprägten Ländern auch im Senegal - gezeigt und online verbreitet wurden, greifen ebenfalls das Thema COVID-19 auf und informieren über Schutzmaßnahmen.

Für Menschen auf der Flucht und in Residenzländern stellen Smartphones häufig die einzigen Möglichkeiten dar, auf Informationen zugreifen zu können. Sie dienen daher nicht nur als Kommunikationsmittel, sondern vor allem auch als Informationsmedium (Richter et al. 2018). Durch das Internet und besonders durch soziale Medien bleiben die Personen über aktuelle Entwicklungen in den Herkunfts- und Aufnahmeländern auf dem Laufenden. Im Kontext der CoronaPandemie bietet das Smartphone Informationen in der jeweiligen Muttersprache. Eine Herausforderung ist jedoch die Bekämpfung von sogenannten 〉Fake News`, der sich lokale und internationale Institutionen wie die Afrikanische Union widmen (Witt 2020).

Eine zusätzliche Möglichkeit der Sensibilisierung sind Corona-Songs, die über die sozialen Medien verbreitet werden und mit Smartphones von überall aufgerufen werden können. Der Wert von Musik als Kommunikationsmittel in Krisen der öffentlichen Gesundheit, insbesondere in Westafrika, wurden etwa im Kontext der Ebola-Epidemie ausführlich untersucht (Tucker 2014; Slaney 2016; McConnell/ Darboe 2017; Rivera 2017; Stone 2017; Deffor 2019). Dabei beleuchten Stone (2017) und Tucker (2014) die globale Verbreitung der Ebola-Songs durch das Internet, sie beschränken sich jedoch auf die mögliche Rezeption der Diaspora und die Bedeutung für das Verständnis der lokalen Entwicklungen durch Außenstehende, die nicht unmittelbar von der Ebola-Epidemie betroffen sind. Das Potenzial solcher Lieder als Sensibilisierungs- und Informationsquelle für Menschen auf der Flucht und in Residenzländern wurde hingegen bisher nicht unter- 
sucht. Eben diesem Aspekt widmet sich der Beitrag anhand senegalesischer Corona-Songs.

\section{Datenmaterial und Analyse}

Zur Analyse konzentriert sich der Artikel auf 20 von insgesamt 36 verfügbaren senegalesischen Corona-Songs, die zwischen dem 18. März und 10. Mai 2020 entstanden sind. Diese Lieder wurden auf Youtube mit den Suchbegriffen »corona songs senegal«, "xeex corona«, »daan corona« sowie der Kombination der Namen bekannter Musiker*innen (Youssou N'dour, Wally Seck, Baaba Maal, Thione Seck, Awadi, Viviane Chidid) mit dem Zusatz »corona« gefunden. 34 dieser Lieder sind hauptsächlich auf Wolof mit wenigen französischen und englischen Passagen, ein Lied ist hauptsächlich auf Poulaar und ein weiteres auf Serer, zwei weniger verbreiteten Sprachen im Senegal. Für eine genauere Analyse wurden die Lieder ausgewählt, die auf Youtube mehr als 25.000 Aufrufe hatten, da davon ausgegangen wird, dass die Zahl der Aufrufe einen Hinweis auf ihre Verbreitung gibt. Am 19. Mai 2020 hatten 21 der Lieder zwischen 25.600 und 781.239 Aufrufe. Hierzu gehört auch das einzige Lied auf Poulaar (Baaba Maal 2020), das aufgrund mangelnder Sprachkenntnisse nicht analysiert werden konnte. Ein weiteres Lied (Bambaly Seck 2020) hat kein Musikvideo, weswegen nur der Text in die Analyse miteinbezogen wird. Insgesamt wurden also 20 Corona-Songs analysiert.

Die senegalesischen Corona-Songs sind hauptsächlich als Bottom-Up-Initiativen entstanden. Lediglich in zwei Fällen haben Musiker*innen direkt mit nationalen Ministerien oder internationalen Organisationen zusammengearbeitet. Die Musiker*innen und Aktivist*innen der Gruppe Y'en a marre, die bereits seit 2011 als regierungskritische Gruppe bekannt ist, suchten beispielsweise gleich nach dem Ausbruch im Senegal den Kontakt mit dem senegalesischen Präsidenten und dem Gesundheitsministerium. Mit den Informationen des Gesundheitsministeriums produzierten sie dann den ersten Corona-Song mit Videoclip, welcher am 19. März 2020 veröffentlich wurde (Feneberg 2020). Baaba Maal (2020) veröffentlichte seinen Corona-Song auf Poulaar in Kooperation mit dem UNCCD, dem Übereinkommen der Vereinten Nationen zur Bekämpfung der Wüstenbildung, für die er als Landbotschafter tätig ist. Auch das senegalesische Gesundheitsministerium veröffentlichte am 19. April 2020 selbst einen Sensibilisierungssong (Ministere de la Santé et de l'action sociale 2020), der allerdings nur sehr selten auf 
Youtube aufgerufen wurde, was dafür spricht, dass er keine weite Verbreitung fand.

Für die Filmanalyse schlägt Mikos die fünf Ebenen von »Inhalt und Repräsentation, Narration und Dramaturgie, Figuren und Akteure, Ästhetik und Gestaltung, Kontexte« vor (Mikos 2015: 43). Die Corona-Songs und die dazugehörigen Videoclips werden in diesem Beitrag mit Fokus auf die Ebenen Inhalt und Repräsentation sowie Ästhetik und Gestaltung analysiert. Ferner wird die Kontextebene berücksichtigt. Durch das Schauen und Hören der 20 ausgewählten, wolofsprachigen Corona-Songs wurden drei Analysekategorien entwickelt: 1) Information über das Coronavirus, 2) Schutzmaßnahmen und Verhaltensregeln und 3) >Fake News<-Dekonstruktion. Diese inhaltliche Analyse wurde dann gleichzeitig mit der Analyse der Videoclips auf ihre Ästhetik und Gestaltung hin durchgeführt.

Anhand einzelner Kommentare zu den Liedern auf Youtube lässt sich nachvollziehen, dass die in diesem Artikel untersuchten senegalesischen Corona-Songs und die dazugehörigen Musikvideos auch in Transit- und Residenzländern rezipiert werden. Die Kommentator*innen befinden sich beispielsweise in Marokko, Mali und Mauretanien sowie in Spanien, Frankreich und Italien. Personen aus Gambia kommentieren ebenfalls, was für eine Verbreitung der Lieder auch unter wolofsprachigen Gambier*innen spricht.

\section{Inhalt senegalesischer Corona-Songs für Geflüchtete und Migrant*innen}

Der erste COVID-19-Fall im Senegal wurde am 2. März 2020 diagnostiziert. Am 15. März 2020 traten die ersten Maßnahmen der Regierung in Kraft wie die Schließung von Schulen, Universitäten und anderen öffentlichen Einrichtungen sowie das Gebot des Social Distancing und der Aufruf, möglichst zu Hause zu bleiben. Am 23. März wurden der nationale Notstand ausgerufen sowie Reisebeschränkungen und eine nächtliche Ausgangssperre verhängt. Nach Inkrafttreten der ersten Maßnahmen sind ab dem 18. März Corona-Songs veröffentlicht worden, die über das Virus und die nötigen Schutzmaßnahmen informieren. Nur $51,9 \%$ der Senegales*innen im Alter von über fünfzehn Jahren sind in der Nationalsprache Französisch alphabetisiert (Human Development Reports 2019). Daher spielen alternative, nicht-schriftliche Informationsquellen und Sensibilisierungsmethoden wie diese Lieder eine große Rolle.

Senegales*innen verlassen das Land aus einer Vielzahl von Gründen. Die Auswirkungen des Klimawandels, die Überfischung des Atlantiks durch ausländische Firmen und die daraus folgenden Schwierigkeiten sowie die weit verbreitete 
Arbeitslosigkeit sind nur einige dieser Gründe (Ziegelmeyer 2014; GierczynskiBocandé 2016). ${ }^{3}$ Laut offiziellen Angaben sind 642.700 senegalesische Migrant*innen sowie weitere 18.200 Personen mit anerkanntem Schutzstatus und 17.400 Personen im Asylverfahren auf der Welt verteilt (IOM, 2020). In Transit- und Residenzländern sind diese Menschen potenzielle Rezipient*innen der Corona-Songs. Hinzu kommen wolofsprachige Gambier*innen, über die es jedoch keine genauen Zahlen gibt.

Lieder zur Sensibilisierung und Aufklärung der Bevölkerung sind im senegalesischen Kontext nichts Neues. Besonders Hip-Hop und Rap sind als Musikgenres bekannt, die politische und gesellschaftliche Missstände anprangern und Veränderungen fordern. Auch während der Präsidentschaftswahlen sind Lieder ein wichtiges Mittel zur Aufklärung über politisch relevante Themen sowie zur Mobilisierung von Wähler*innen. Im Senegal existiert also eine Tradition der Kritik und Information durch Musik (Herson 2010; Appert 2016). Musiker*innen und ihre Lieder sind als vertrauenswürdige Informationsquelle in der Gesellschaft etabliert. Bereits im Kontext der Ebola-Epidemie sind im Senegal Songs zur Sensibilisierung der Bevölkerung entstanden. Daher ist es nicht verwunderlich, dass auch während der Corona-Pandemie diverse Lieder komponiert werden.

\subsection{Informationen über das Coronavirus}

Die untersuchten Lieder bieten korrekte Informationen über COVID-19, die Ausbreitung und Gefahren. Sie beziehen sich sowohl auf die globalen als auch lokalen Entwicklungen und sprechen somit wolofsprachige Menschen unabhängig vom Aufenthaltsort an. Die Songs betonen stets, dass sich das Coronavirus auf der ganzen Welt verbreitet, für Chaos sorgt, sich Menschen täglich anstecken und an den Folgen sterben. Dies wird in den Musikvideos visuell vorrangig durch Clips über Krankenhäuser, Ärzt*innen und Patient*innen weltweit verdeutlicht. Gleichzeitig wird herausgestellt, dass das Virus auch im Senegal angekommen ist und so das lokale Geschehen global eingebettet.

Der Hinweis auf die Verbreitung des Virus weltweit wie auch im Senegal und die Darstellung der Folgen dienen dazu, der Notwendigkeit des Ergreifens von lokalen Schutzmaßnahmen Nachdruck zu verleihen. Das am 19. März 2020 veröffentlichte Lied »Fagaru Ci Coronavirus« vom Zusammenschluss der Musiker*innen und Aktivist*innen der Bewegung Y'en a marre bringt dies in einem

3 Auf Wolof wird das Verlassen des Landes jedoch nicht als Fliehen, sondern als `tukki< (dt. >reisen $\triangleleft)$ bezeichnet. Eine Senegales*in im Ausland ist demnach ein*e `tukkikat $($ dt. $>$ Reisende*r $\triangleleft)$. 
Slogan auf den Punkt: »Fagaru gënn faju«, was übersetzt »Vorsorgen ist besser als ärztlich behandelt zu werden « bedeutet. Y'en a marre hat es mit dieser Wendung geschafft, den Nutzen der Vorsorgemaßnahmen in einem griffigen, eingängigen Satz zu erfassen. Viele der danach veröffentlichten Lieder übernehmen diesen Slogan in ähnlicher Form. Die Gruppe X'Press verwendet in ihrem am 27. März 2020 veröffentlichten Song »Fagaru (Si Coronavirus Covid-19)« beispielsweise sogar exakt denselben Slogan. Da Y'en a marre den Corona-Song bereits sehr früh veröffentlichten, haben sie sicher eine gewisse Vorbildfunktion für andere Musiker*innen. Weil Y'en a marre mit dem Gesundheitsministerium kooperierte, sind die Informationen über das Virus und die Schutzmaßnahmen staatlich autorisiert, sodass auch Nachahmer*innen amtliche Informationen verbreiten.

In allen Liedern wird nachdrücklich betont, dass nur das Einhalten der Verhaltensregeln zuverlässigen Schutz vor dem Coronavirus bietet sowie die Pandemie kontrollieren und beenden lässt. Das Fehlen einer Impfung und eines zur Bekämpfung der Krankheit zugelassenen Medikaments wird erläutert. Zugleich unterstreichen die Songs, dass die Maßnahmen zu beachten sind, um nach der Bekämpfung der Pandemie den Normalzustand erreichen zu können. Damit einhergehend ist die hohe Ansteckungsgefahr ein zentrales Thema. Ein Großteil der Lieder erklärt die Ansteckungswege des Virus und beleuchtet die Übertragung durch Tröpfchen- und Schmierinfektionen sowie durch Aerosole. Wichtig ist, dass medizinische Informationen in einfachen Worten erklärt und häufig durch Animationen im Clip visualisiert werden. Das vereinfacht das Verständnis über die komplexen Gefahren. Viele Lieder zählen zudem die möglichen Symptome (Fieber, Halsschmerzen, Kopfschmerzen, Gliederschmerzen, Müdigkeit/Erschöpfung, Schüttelfrost, Husten, Atemwegsbeschwerden) auf und betonen die Ähnlichkeit mit Grippesymptomen.

Auf besonders eindringliche Art thematisieren alle untersuchten Corona-Songs die Letalität des Virus. Der Fokus der Lieder liegt darauf, dass das Virus potenziell jeden bedrohen kann und nicht nur besonders vulnerable Gruppen wie ältere oder vorerkrankte Menschen. So erwähnen etwa einige Lieder die bisherigen prominenten COVID-19-Todesopfer wie den kamerunischen Musiker Manu Dibango und den senegalesischen Fußballfunktionär Pape Diouf (z.B. Queen Biz 2020; Demba Guisse et al. 2020; Dior Mbaye 2020). Die Gefährlichkeit des Virus wird durch eine sehr starke, an Krieg erinnernde Rhetorik in den Texten unterstrichen. Dip Doundou Guiss (2020) spricht etwa von Ärzt*innen an der Front und Y'en a marre (2020) davon, dass das Virus Verwüstungen auf der ganzen Welt anrichtet. Die Sänger*innen Dior Mbaye (2020), Sokhou BB (2020) und Queen Biz (2020) verstärken diese Kriegsrhetorik in ihren drei Corona-Songs durch eine gewisse 
Dramatik in der Stimme und durch ihr Gebaren in den Musikvideos. Auch der Titel des Songs »Daan Corona« (Youssou Ndour et al. 2020), auf Deutsch »Corona bezwingen« rekurriert auf das kriegerische Bezwingen eines Feindes. In den Corona-Songs dienen folglich sprachliche wie auch musikalische Mittel der Betonung der Dringlichkeit von Botschaften.

Insgesamt lässt sich festhalten, dass die Corona-Songs zuverlässig über die Verbreitung des Virus weltweit und im Senegal informieren. Die Ansteckungswege, die möglichen Symptome und besonders gefährdete Gruppen werden erläutert. Die vom Virus ausgehende Gefahr wird hervorgehoben: jede*r kann sich anstecken und infolgedessen auch sterben. Diese Information ist auch für Migrant*innen und Geflüchtete von großer Relevanz.

\subsection{Schutzmaßnahmen und Verhaltensregeln}

Alle Corona-Songs erwähnen in unterschiedlichem Maße Schutzmaßnahmen wie das Händewaschen mit Seife, das Tragen von Masken, das Husten in die Armbeuge und die Verwendung von Desinfektionsmitteln. Auch die Verhaltensregeln, wie auf den Handschlag und Umarmungen zu verzichten, zu Hause zu bleiben, Versammlungen zu meiden und Social Distancing generell einzuhalten, finden Einzug in die Lieder. Häufig werden die Maßnahmen in den Musikvideos passend zum Text visualisiert. Im Video zum Corona-Song von Dip Doundou Guiss (2020) werden etwa einzelne zu Hause aufgenommene Clips von verschiedenen Personen gezeigt, die die Schutzmaßnahmen praktisch durchführen (z.B. Hände mit Seife waschen). Im Musikvideo des Songs »Stop Corona" von Cherifou \& Job sa brain et al. (2020) stellen die Musiker*innen einzelne Berufsgruppen wie Schneider*in, Elektriker*in, Maler*in, Krankenpfleger*in und Eierverkäufer*in dar. Dadurch verdeutlichen sie, dass alle Menschen vom Coronavirus betroffen sein könnten und daher auch alle die Schutzmaßnahmen und Verhaltensregeln respektieren müssen.

In Wally B. Secks (2020) Musikvideo verleiht der Playbackgesang berühmter senegalesischer Persönlichkeiten, beispielsweise Politiker*innen und Fernsehmoderator*innen, der Wichtigkeit der Einhaltung der Schutzmaßnahmen Nachdruck. Das Vertrauen in die verbreiteten Informationen wird dadurch erhöht. Dass zum Schluss selbst der Kulturminister spricht und das Engagement des Sängers lobt, verdeutlicht die politische Unterstützung der musikalischen Aufklärung über das Coronavirus. Die Bottom-Up-Initiative der einzelnen Musiker*innen finden hier Einzug in die amtliche Strategie der Bekämpfung des Coronavirus. 
Die kollektive Ebene spielt in den Corona-Songs eine große Rolle. Es wird betont, dass die Pandemie nur bewältigt werden kann, wenn alle Menschen zusammenarbeiten. In den Liedern wird zu Solidarität und Zusammenarbeit aufgerufen. Der Slogan und Titel eines vom Journal Rappé (2020) veröffentlichten Kollaborationssongs "And xeex corona«, auf Deutsch "zusammen Corona bekämpfen«, signalisiert das. Auch die Kollaborationen verschiedener senegalesischen Musiker*innen veranschaulicht gelebte Zusammenarbeit. Doch nicht nur der Zusammenhalt in der senegalesischen Gesellschaft, sondern auch der globale Zusammenhalt wird beschworen. Geflüchtete und Migrant*innen können sich durch die Einbettung in das globale Kollektiv als Teil der Anstrengungen zur Bewältigung der Pandemie fühlen. Die globale Solidarität wird jedoch nicht immer ohne Zynismus erwähnt. Dior Mbaye (2020) bedauert in ihrem Lied »En bas Coronavirus« (dt. »Weg mit dem Coronavirus«), dass es für ein globales Bewusstsein und weltweite Solidarität ein Coronavirus braucht. Sie kritisiert damit den Fokus vieler Menschen auf den Nationalstaat, durch den globale Probleme in Zeiten ohne Pandemie hintenangestellt werden.

Auch der Aufruf zu Solidarität und Mitgefühl mit bereits infizierten Personen bezieht die nationale und internationale Ebene mit ein. Y'en a marre (2020) sprechen sich etwa deutlich gegen die Diskriminierung von Infizierten aus. Gleichzeitig findet der Dank an das medizinische Personal in mehreren Liedern seinen Platz (z.B. durch Cherifou \& Job sa brain et al. 2020, Clayton Hamilton et al. 2020). Im vom Journal Rappé (2020) produzierten Song wird dazu aufgerufen, für die Opfer der Corona-Pandemie zu beten. Auch in weiteren Liedern wird zum Gebet aufgefordert, um die Pandemie gut zu bewältigen (z.B. durch X-Press 2020, Kine Laam et al. 2020). Hier lassen sich klare Rückschlüsse auf Bewältigungsstrategien von Geflüchteten und Migrant*innen in Transit- und Residenzländern ziehen. Wissenschaftliche Studien unterstreichen die hohe Bedeutung von (religiösem) Glauben an eine bessere Zukunft als Antriebskraft für den Umgang mit schwierigen Lebensumständen (Ager/Fiddian-Qasmiyeh/Ager 2015; Krause 2016). Gerade für Geflüchtete und Migrant*innen, die sich aufgrund einer fehlenden Gesundheitsversorgung sowie der nötigen Infrastruktur zur Einhaltung der Schutzmaßnahmen in einer besonders vulnerablen Situation befinden, können Gebet und Glaube eine wichtige Rolle zur Bewältigung der Corona-Pandemie spielen. Mit der Thematisierung ist anzunehmen, dass die Corona-Songs diese wichtigen Bewältigungsstrategie befördern und Identifikationspotenzial für Menschen in Transit- und Residenzländern bieten können.

Schließlich erfüllen die Corona-Songs die Funktion des »Edutainment« (Deffor 2019), also der Verbreitung verlässlicher Informationen in einem ansprechenden 
und einprägsamen Format. Die dargestellten Informationen über das Coronavirus und die zu beachtenden Maßnahmen sind sowohl für die Bevölkerung als auch für Migrant*innen und Geflüchtete von großem Nutzen. Der Fokus der Lieder liegt auf den generell geltenden Maßnahmen wie »Tóogleen sen kër« (dt. »Bleibt zu Hause «) und den Hygieneregeln. Einzig die Telefonnummern (spezielle COVID-19-Hotline »numéro vert" und die allgemeine Notrufnummer), welche im Falle des Auftretens von COVID-19-Symptomen angerufen werden sollen, sind spezifisch für den Senegal. Migrant*innen und Geflüchtete können zwar außerhalb des Senegals die spezielle Nummer nicht anrufen, sie können hiervon aber ableiten, dass Krankenhäuser oder Praxen beim Verdacht einer COVID-19Infektion vor dem Aufsuchen informiert werden müssen. Weitere staatliche Maßnahmen wie zum Beispiel die nächtliche Ausgangssperre im Senegal finden keine Erwähnung in die Corona-Songs.

\subsection{Dekonstruktion von 'Fake Newsı}

Alle Corona-Songs betonen, dass das Virus wirklich existiert und gefährlich ist. Sie positionieren sich deutlich gegen die Leugnung des Vorhandenseins des Virus und weiterer `Fake News〈. Das Lied »Corona Virus« von Bitikou Laye ft. Esprit (2020) soll hier beispielhaft für eine Analyse der Dekonstruktion von Falschinformationen über das Coronavirus dienen.

Das Video spielt in einer >Bitikı (Wolof für das frz. Wort >boutiqueく, auf Deutsch `Tante-Emma-Laden (). Bitikou Laye spielt den Verkäufer, der mit Maske und Handschuhen hinter dem Verkaufstresen steht, während Esprit als Kunde ohne Schutz auftritt. Sie führen ein Gespräch in Liedform und diskutieren über das Coronavirus, die Schutzmaßnahmen sowie die Verhaltensregeln. Esprit glaubt nicht an die Existenz des Coronavirus und hält die von Bitikou Laye ergriffenen Schutzmaßnahmen für verrückt. Beide sprechen über die Tödlichkeit des Virus, wobei Bitikou Laye insistiert, dass sowohl starke Menschen als auch Kinder durch das Virus sterben können. Der Kunde hingegen bezweifelt, dass ein Virus ihn töten könne. Die Wichtigkeit des Tragens von Masken, von Desinfektionsmitteln und des Verzichts auf den Handschlag werden von Bitikou Laye angeführt. Er unterstreicht außerdem, dass die Hinweise der Ärzt*innen befolgt werden müssten. Esprit jedoch glaubt an die Kraft des Gebets, durch welches er gesund bleiben wird. Der Glaube dient also nicht nur als Bewältigungsstrategie in der Pandemie, sondern kann auch instrumentalisiert werden, um den Verhaltensregeln nicht zu folgen. Das Gespräch wird durch das Klingeln von Esprits Telefon unter- 
brochen. Ein Freund ruft ihn an und berichtet ihm von der Infektion eines Bekannten mit dem Coronavirus. Esprit reagiert geschockt. Eine dritte Person tritt in den Laden, ebenfalls ohne Maske, und möchte den anderen die Hand geben. Esprit schreckt vor seiner Hand zurück und entschuldigt sich, dass er aufgrund des Virus nicht die Hand gibt. Er verlässt schnell die >Bitikı und Bitikou Laye zeigt sich von seinem plötzlichen Sinneswandel überrascht.

Die im Musikvideo dargestellte Situation erscheint alltäglich, eingängig und allgemein verständlich. Menschen dürften sich also gut mit der Situation und den Personen identifizieren können. Der Laden als Schauplatz des Videos und die Interaktion zwischen Verkäufer und Käufer sind zudem nicht spezifisch für den Senegal, wodurch sich ein großes Identifikationspotenzial für Menschen in Transit- und Residenzländern ergibt.

Auch andere Corona-Songs widmen sich durch direkte und indirekte Kritik der Dekonstruktion von >Fake News $<$. Dip Doundou Guiss (2020) bezeichnet etwa Menschen als Lügner*innen, die behaupten, dass schwarze Menschen nicht an COVID-19 erkranken könnten. Andere Künstler*innen >beweisen diesen Fakt durch das Anführen des Todes von schwarzen Prominenten (siehe oben). Kine Laam et al. (2020) widmen sich sogar internationalen >Fake News der eigenständigen Einnahme des Malariamedikaments Chloroquin ohne ärztliche Verordnung ab, welches vom US-amerikanischen Präsidenten Donald Trump als Vorsorge empfohlen wurde (dpa 2020). Wolofsprachige Menschen in den USA können durch diesen Song folglich zusätzliche Informationen zu ihrem Schutz erhalten, was die potenzielle Reichweite der Corona-Songs und ihre mögliche Bedeutung als Informationsquelle für Migrant*innen und Geflüchtete außerhalb des Senegals verdeutlicht.

\subsection{Visualisierung der Inhalte in den Musikvideos}

Abgesehen von der bereits erwähnten Funktion der Musikvideos, den Schutzmaßnahmen und Verhaltensregeln durch passende Bilder Nachdruck zu verleihen, fallen zwei weitere Punkte auf: erstens die Kooperation mit anderen Musiker*innen und Figuren des öffentlichen Lebens und die besondere Ästhetik der dazugehörigen Videoclips sowie zweitens die Präsenz von Bildern, die auf die Corona-Pandemie im Globalen Norden verweisen.

Sechs der analysierten 20 Lieder sind Kooperationen von senegalesischen Musiker*innen auf musikalischer und visueller Ebene. Die Musikvideos dieser Songs erinnern stark an die Ästhetik von »We are the World«, einem 1985 von 
einem Zusammenschluss internationaler Musiker*innen namens U.S.A. for Africa produzierten Song, oder ähnlichen Songs, die nach Katastrophen im Globalen Süden zum Spenden animieren sollen (vgl. Fayoyin und Nieuwoudt 2017). Diese Gestaltung der Musikvideos verbildlicht, dass die Corona-Pandemie als Katastrophe wahrgenommen wird. Die Musiker*innen werden im Studio beim Aufnehmen des Songs gefilmt und lesen teilweise ihre Texte ab. Hierdurch wird die Dringlichkeit der Botschaft betont, da die Videos und Lieder innerhalb kürzester Zeit aufgenommen erscheinen. In den Videoclips werden außerdem aus Nachrichtensendungen entnommene Aufnahmen der katastrophalen Zustände hauptsächlich in europäischen Krankenhäusern auf dem Höhepunkt der Pandemie gezeigt. Clips der senegalesischen oder anderer afrikanischer Krankenhäuser sind selten. Dies ist von besonderem Interesse, denn in der Regel sind in Musikvideos mit dieser Ästhetik Bilder von Katastrophen im Globalen Süden zu sehen. Bilder von Seuchen in Europa, weißen Ärzt*innen und Krankenpfleger*innen in Schutzanzügen und weißen Patient*innen und afrikanische Musiker*innen, die diese globale Katastrophe besingen, sind eine Neuheit. Es findet eine Aneignung der $»$ We are the World«-Ästhetik durch die senegalesischen Künstler*innen statt, die die globalen Machtverhältnisse in Frage stellt.

Nicht nur in den Videos der Kooperations-Corona-Songs finden sich Clips, die die Auswirkungen der Pandemie im Globalen Norden zeigen. Auch in anderen Liedern werden immer wieder ähnliche Aufnahmen gezeigt. Besonders drastisch sind die Bilder von vielen nebeneinanderstehenden Särgen in Norditalien, die in verschiedenen Videos enthalten sind. Diese Bilder sollen die Schwere der Pandemie verdeutlichen, legen aber gleichzeitig den Fokus auf ihre globalen Auswirkungen. Für Geflüchtete und Migrant*innen sind sie von besonderer Bedeutung, da sie Situationen zeigen, die in ihren Transit- und Residenzländern vorkommen. Dadurch wird die Gefahr des Coronavirus verdeutlicht und gezeigt, dass die Verhaltensregeln und Schutzmaßnahmen nicht nur für den senegalesischen Kontext gelten, sondern auch in anderen Ländern eingehalten werden müssen. Dies unterstreicht die thematische Relevanz der Corona-Songs außerhalb des Senegals.

\section{Fazit}

Aufgrund der Vermittlung von Informationen über das Virus wie auch über die zur Vermeidung einer Infektion nötigen Schutzmaßnahmen und Verhaltensregeln erweisen sich die senegalesischen Corona-Songs als eine verlässliche Informationsquelle. Ein Großteil dieser Informationen über Maßnahmen und Regeln sind 
nicht spezifisch für den senegalesischen Kontext, sondern generell gültig. Hierdurch werden die Songs auch für wolofsprachige Migrant*innen und Geflüchtete relevant. Sie können auf die Corona-Songs zurückgreifen, um die notwendigen Informationen zu erhalten. Der Inhalt der Songs ist einprägsam und leicht verständlich und wird durch Bilder und Clips in den Musikvideos auch visuell fassbar gemacht. Die Songs greifen außerdem Falschinformationen auf und dekonstruieren sie. Damit bilden sie ein wichtiges Gegengewicht zu über Social-MediaKanäle verbreiteten >Fake News` und bieten eine wichtige Möglichkeit des Zugangs zu verständlichen Informationen in der Pandemie.

Die Musiker*innen gehen in den Corona-Songs allerdings nicht auf die Schwierigkeiten der Durchführbarkeit der vorgeschlagenen Maßnahmen ein. Sowohl die Bevölkerung im Senegal als auch senegalesische Geflüchtete und Migrant*innen in Transit- und Residenzländern stehen aufgrund der mangelnden Infrastruktur - wie beispielsweise dem Zugang zu Wasser und Seife - bei der Einhaltung der Hygienemaßnahmen vor großen Herausforderungen. Die Songs beschränken sich stattdessen auf eine Sensibilisierungsfunktion und auf die Bereitstellung von wichtigen Informationen zur Corona-Pandemie. Dies ist zwar in der aktuellen Situation sehr wichtig. Die Frage, warum die Musiker*innen generell und gerade regierungskritische Gruppen wie Y'en a marre diese praktischen Herausforderungen nicht in ihre Lieder aufnehmen, bleibt jedoch unbeantwortet und bedarf weiterer Forschung. Es bleibt abzuwarten, ob im Nachgang des akuten Pandemiegeschehens kritischere Songs veröffentlicht werden, die sich mit den sozialen, ökonomischen und politischen Folgen der Maßnahmen auseinandersetzen. Auch die Rezeption der aktuellen Corona-Songs durch die senegalesische Bevölkerung sowie durch Migrant*innen und Geflüchtete sollte in einem zweiten Schritt untersucht werden, um die Wirkung der Lieder zu analysieren.

\section{Korpus der Corona-Songs}

Baaba Maal (2020), »Jam Leeli Jam Yo«, https://www.youtube.com/watch?v=n0 QB-lmHwvw, 19.5.2020.

Bambaly Seck (2020), »Corona«, https://www.youtube.com/watch?v=uiIgzrFhcv 8, 19. Mai 2020.

Boutikou Laye feat. Esprit (2020), »Corona Virus «. https://www.youtube.com/wa tch?v=Ge7NzY_F4Fo, 19.5.2020.

Bril x Bass Thioung (2020), »Noy Moyto Corona«, https://www.youtube.com/wa tch? $=$ R00bRWo84mY, 19.5.2020. 
Cherifou \& Job sa brain/Dieyla/Keur gui family/Hamdy/Papa Laye (2020), »Stop

Corona «, https://www.youtube.com/watch?v=5EAo8rgBVp4, 19.5.2020.

Clayton Hamilton/Awadi/Ombre Zion/Dame Sene/Bakhaw/Titi/Ngaaka Blindé/

Adiouza/Fata EL Présidenté/Matadi/Dahman Pro/Gougou (2020), »Covid 19«, https://www.youtube.com/watch?v=1b5kHnHznh4, 19.5.2020.

Demba Guisse/Talla Diazaka/Pendo Guisse/Bocar Kora/Thiate Seck/Mame

Coumba Mbaye/Alé Dieng/Diaw Diop/Killer X/Clayton Hamilton (2020), »Mouslou si Corona«, https://www.youtube.com/watch?v=MlBkfpXN O8k, 19.5.2020.

Dior Mbaye. (2020), »En bas Coronavirus«, https://www.youtube.com/watch?v= Bs hrnxkWJM, 19.5.2020.

Dip Doundou Guiss (2020), »And xeex Corona«, https://www.youtube.com/watc $\mathrm{h} ? \mathrm{v}=\mathrm{dwD} 0 \mathrm{rz63SSA}, 1$ 19.5.2020.

Journal Rappé (2020), »NA FI JOGGE (AND XEEX CORONA)«, https://www.y outube.com/watch? $\mathrm{v}=$ EaHMbyAjCFc, 19.5.2020.

Kine Laam/Fatou Laobe/Sode Mama Fall/Amy Colé Dieng/Mamy Victory/Ndiolé Tall/Idrissa Diop/Daba Seye (2020), »Compil Covid19 Senegal Super Ndanane Deluxe«, https://www.youtube.com/watch?v=VbzVglFv8II, 19.5.2020.

Maabo (2020), »Stop Corona«, https://www.youtube.com/watch?v=Ua9BI91JPH A, 19. Mai 2020.

MINISTERE DE LA SANTE ET DE L'ACTION SOCIALE (2020), »COVI-

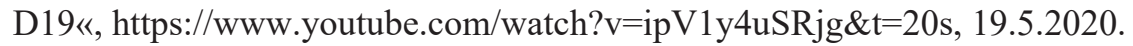

One Lyrical (2020), »Covid 19 (Joro Remix)«, https://www.youtube.com/watch?v $=\mathrm{kb} 8$ Or6-WgwA, 19.5.2020.

Queen Biz (2020), »Corona«, https://www.youtube.com/watch?v=VHPnL1gDlJg, 19.5.2020.

Sokhou BB (2020), »Daan Covid 19«, https://www.youtube.com/watch?v=qFW5 F61aMQ8, 19.5.2020.

Thione Ballago Seck (2020), »Corona«, https://www.youtube.com/watch?v=NTO fcLc5bV8, 19.5.2020.

Wally B. Seck (2020), »Digglé«, https://www.youtube.com/watch?v=3mKpYFhh 9ps, 19.5.2020.

X-Press (2020), »Fagaru (Si Coronavirus Covid-19)«, https://www.youtube.com/ watch? $\mathrm{v}=\mathrm{LpUQpGWuYyU,} \mathrm{19.5.2020.}$

Y'en a marre (2020), »Fagaru Ci Coronavirus«, https://www.youtube.com/watch? $\mathrm{v}=06 \mathrm{YbY} 1 \mathrm{MLp} 4 \mathrm{~A}, 1$ 19.5.2020. 
Youssou Ndour/Awadi/Matador/Mamy Victory/Duggy Tee/Baxaw/ Simon/OMG/Dip Doundou Guiss/Samba Peuzzi/Korka Dieng/Moonaya/ Xuman/Ngaaka Blinde/Ndongo D/Clayton Hamilton/Viviane/Pape Birahim/Fou Malade/Idrissa Diop (2020), »Daan Corona«. https://www.youtu be.com/watch?v=qwT19-up78M, 19.5.2020.

Zbest Family (2020), »Coronavirus«, https://www.youtube.com/watch?v=Dhahfu RBwLo, 19.5.2020.

\section{Literatur}

Ager, Joey/Fiddian-Qasmiyeh, Elena/Ager (2015), Local Faith Communities and the Promotion of Resilience in Contexts of Humanitarian Crisis, Journal of Refugee Studies, 28 (2), 202-221.

Appert, Catherine M. (2016), Locating Hip Hop Origins: Popular Music and Tradition in Senegal, Africa, 86 (2), 237-62.

Casey, Ruairi (2020), In vast Kenya Camp, Refugee Journalists on Coronavirus Front line, aljazeera.com, 27. Mai 2020, https://www.aljazeera.com/news/202 0/05/vast-kenya-camp-refugee-journalists-coronavirus-front-line-2005251023 45033.html.

Deffor, Sally (2019), Ebola and the Reimagining of Health Communication in Liberia, in: Tangwa, Godfrey B., et al. (Hrsg.), Socio-cultural Dimensions of Emerging Infectious Diseases in Africa. An Indigenous Response to Deadly Epidemics, Cham, 109-121.

Doctors of the World/British Red Cross (2020), Gindikaayu Coronavirus (Covid19).

dpa (2020), Trump nimmt Malaria-Medikament zur Corona-Vorbeugung, tagesspiegel.de, 19. Mai 2020, https://www.tagesspiegel.de/politik/experten-warnen -vor-angeblichem-wundermittel-trump-nimmt-malaria-medikament-zur-coron a-vorbeugung/25842068.html.

Fayoyin, Adebayo/Nieuwoudt, Susan (2017), The Power of Song in the Struggle for Health and Development Outcomes in Africa: Lessons for Social and Behaviour Change Programmes, Journal of Mass Communication and Journalism, 7 (4), 1-6.

Fischer, Jonathan (2020), Wenn beten nicht reicht, SZ.de, 30. März 2020, https:// www.sueddeutsche.de/kultur/afrika-wenn-beten-nicht-reicht-1.4861889.

Feneberg, Valentin (2020)〉 »Wir leisten Aufklärung«, taz, 24. Mai 2020, https://ta z.de/Rap-Aktivist-ueber-Corona-in-Senegal/!5687158/. 
Gierczynski-Bocandé, Ute (2016), Senegal: das Tor Westafrikas zwischen Immigration und Emigration, ET-Studies, 7 (2), 245-260.

Hargreaves, Sally et al. (2020), Europe's Migrant Containment Policies threaten the Response to Covid-19, British Medical Journal, 368 (March), 1-2.

Herson, Ben (2010), A Historical Analysis of Hip-Hop's Influence in Dakar from 1984-2000, American Behavioral Scientist, 55 (1), 24-35.

Høvring, Roald (2020), 10 Things You Should Know About Coronavirus and Refugees, The Norwegian Refugee Council, 16. März 2020, https://www.nrc.n o/news/2020/march/10-things-you-should-know-about-coronavirus-and-refug ees/.

Human Development Reports (2019), Human Development Indicators Senegal, UNDP, http://hdr.undp.org/en/countries/profiles/SEN, 1.7.2020.

IOM (2020), Migration Data Portal Senegal, 2020, https://migrationdataportal.org $/$ ? $\mathrm{i}=$ stock_abs_origin\& $\mathrm{t}=2019 \& \mathrm{~cm} 49=686,1.7 .2020$.

Krause, Ulrike (2016), Wie bewältigen Flüchtlinge die Lebensbedingungen in Flüchtlingslagern? Ergebnisse aus einer empirischen Analyse zu kongolesischen Flüchtlingen in Uganda, Zeitschrift für Friedens- und Konfliktforschung, 5 (2), 189-220.

McConnell, Bonnie B./Darboe, Buba (2017), Music and the Ecology of Fear: Kanyeleng Women Performers and Ebola Prvention in The Gambia, Africa Today, 63 (3), 29-42.

Mikos, Lothar (2015), Film- und Fernsehanalyse, Konstanz/München.

Refugees International (2020), COVID-19 and the Displaced: Addressing the Threat of the Novel Coronavirus in Humanitarian Emergencies, 1-26, https:// www.refugeesinternational.org/reports/2020/3/29/covid-19-and-the-displacedaddressing-the-threat-of-the-novel-coronavirus-in-humanitarian-emergencies, 1.7.2020.

Richter, Carola/Emmer, Martin/Kunst, Marlene (2018), Von Smartphones, Informationsnetzwerken und Misstrauen - Mediennutzung im Kontext von Flucht, Zeitschrift für Flüchtlingsforschung, 2 (2), 292-312.

Rivera, Michael (2017), Music, Media, and the Ethnopoetics of Two Ebola Songs in Liberia, Africa Today, 63 (3), 63-76.

Slaney, Evan (2016), Socratic Dialogue as a Framework for Understanding Activist Music during the Ebola Outbreak in Liberia, Nota Bene: Canadian Undergraduate Journal of Musicology, 9 (1), 71-86.

Stone, Ruth M. (2017), >Ebola in Town «: Creating Musical Connections in Liberian Communities during the 2014 Crisis in West Africa, Africa Today, 63 (3), 79-97. 
Tucker, Boima (2014), Beats, Rhymes and Ebola, Fieldsights - Hot Spots, Cultural Anthropology Online, http://www.culanth.org/fieldsights/beats-rhymes-and -ebola.

Uimonen, Paula (2020), Better Day. Covid-19 Music Video of Hope and Care from Tanzania, Antroperspektiv, https://antroperspektiv.org/2020/04/28/betterday-covid-19-music-video-of-hope-and-care-from-tanzania/.

UNESCO (2018), Global Education Monitoring Report 2019. Migration, displacement and education: Building bridges, not walls, Paris.

UNHCR (2020), COVID-19 Emergency Response Update. West \& Central Africa, UNHCR, https://reporting.unhcr.org/sites/default/files/EXTERNAL \%2 0UNHCR\%20RBWCA\%20-\%20COVID19\%20Regional\%20SitRep\%20-\%2 020200701.pdf, 1.7.2020.

United Nations (2020), Policy Brief: COVID-19 and People on the Move, https:// unsdg.un.org/sites/default/files/2020-06/SG-Policy-Brief-on-People-on-the-M ove.pdf, 1.7.2020.

Witt, Antonia (2020), An Island of Internationalism: The African Union's Fight Against Corona, PRIF BLOG, https://blog.prif.org/2020/04/07/an-island-of-int ernationalism-the-african-unions-fight-against-corona/,1.7.2020.

Ziegelmeyer, Usha (2014), >Wir sind hier, weil ihr unsere Länder zerstört`, Böll Thema, 3 (2), 8-9.

\section{Autorin}

Julia Stier, M.A., Wissenschaftszentrum Berlin für Sozialforschung (WZB), Berlin 